\title{
The SMA properties in civil engineering applications. The SMARTeR project: Use of SMA in damping of stayed cables for bridges
}

\author{
V. Torra ${ }^{\mathrm{a}}$, A. Isalgue, G. Carreras, F.C. Lovey ${ }^{1}$, H. Soul ${ }^{1}$, P. Terriault ${ }^{2}$, B. Zapico ${ }^{3}$ \\ CIRG-Applied Physics, ETSECCPB, UPC, Barcelona, Catalonia, E-08034 Spain \\ ${ }^{1}$ Centro Atomico Bariloche (CAB-IB) Bariloche, 8400 Argentina \\ ${ }^{2}$ LAMSI, ETS, Université de Québec, H3C 1K3 Montréal, Ca. \\ ${ }^{3}$ ELSA Lab. Joint Research Center - EU, Ispra, 21020 (VA)-Italy
}

\begin{abstract}
One of the classical applications of the SMA is based on the used of the hysteresis cycle associated to the martensitic transformation (classically, described as a first order phase transition) for damping devices. For each application a detailed knowledge of the conditions to be accomplished by the material is absolutely necessary. For the stayed cables in bridges of the Iroise bridge, the oscillation frequencies are 1 and $3 \mathrm{~Hz}$; and the peak to peak oscillation amplitude is close to $10 \mathrm{~cm}$ and, also, the SMA works in close contact with the external ambient temperature. In fact, the bridge is a $2 * 2$ lanes free highway situated between Brest and Plougastel (France). The main requirement is related to several days of intense winds or rain associated to strong storms. The conditions imposed to the samples suggest appropriate behavior for, at least, 500000 cycles of working. The experimental analysis, centered in $\mathrm{NiTi}$, is focused in 1) the fatigue life of the samples, 2) the evaluation of the Clausius-Clapeyron coefficient, 3) assuring that the degradation of the material properties remains below a safety limit in the expected time scale of application, 4) modeling the cable behavior to show the positive damping capacity of SMA and 5) studying the applications in semi-realistic scale in the ELSA-JRC (a civil engineering facility in Ispra, Italy) demonstrating the positive effect of the SMA.
\end{abstract}

\section{Introduction}

The problems related with the search of an effective mitigation of the actions of the storms (i.e., wind, rain and/or traffic) that induces oscillations in stayed cables for bridges are of permanent interest to increase the safety and life of the bridges. Recently, the SMA's were proposed as useful passive devices for dampers in Civil Engineering [see ref 1 and related references]. The SMAs show a hysteresis due to their martensitic transformation (a called first order phase transformation of "military" characteristics). In SMA the damping effect arise from the absorption of mechanical work and their transformation in heat in the SMA through the hysteresis cycle. The self-heating is intrinsic to damping effect and need to be considered in relation with the cycling amplitudes and frequencies. In addition, for each application, a careful analysis of their explicit (and, also implicit) requirements is very important to achieve an appropriate guaranteed behavior of the material. For instance, one macroscopic or mesoscopic property like the hysteresis cycle evolves on cycling and the origins of the evolution may be associated to plastic deformation, interaction between the interface corresponding to phase transition with the grain boundaries, precipitates and/or other defects.

Furthermore, atomic diffusion, some times partially recoverable, may change the hysteresis width and the Ms [2]. Moreover, a spontaneous increase of length of the sample with cycling: the SMA creep needs to remain under control to avoid loses of damping due to shortening of the transformation length. The requirements for the SMA in damping stayed cables in bridges oscillating under the principal action of wind/rain come from the number of oscillation cycles occurring in 3 or 4 days in a strong storm. The number of cycles at $1 \mathrm{~Hz}$ overcomes 500000 at lower deformation (under 2-3\%). In addition it is wanted that some pause between sets of cycles would not induce changes in the hysteresis. In general, it is necessary that the SMA would be able work continuously, either in summer or in winter with satisfactory performance. For instance, in winter, the damper could remain in martensite without retransformation to parent phase with disappearance of the damping effect, or in summer the increase of stress associate to Clausius-Clapeyron coefficient could reduce the number of working cycles to fracture. The required fatigue-life is extremely higher in comparison with the application in earthquake damping. The eventual use of pre-stressed material always provides a better behavior.

\footnotetext{
${ }^{a}$ e-mail : vtorra@fa.upc.edu
} 
The mechanical analysis establishes that the use of pre-stressed dampers shows a path of the hysteresis cycle that includes more area in force-deformation representation and the conversion of mechanical energy to heat is more efficient than trajectories starting in elastic part. In fact, the elastic or reversible part is partially avoided. Use of pre-stressed materials is not appropriate for $\mathrm{Cu}$-based alloys due to the martensite stabilization. But, for damping of stayed cables the motionless time to start, previous to wind/storms that induce oscillations are short (some days/months) in comparison with the eventual long time required for one earthquake (several years/decades). Usually the length of the specimens increases progressively with cycling (i. e., the SMA creep). As, in fact, the behavior of the damper requires some fixed length of the damper used, the applicability of the SMA requires appropriate thermomechanical study of this behavior in order to reduce or suppress the spontaneous increase of length. The origin of SMA creep is a particular property of each alloy. In Cu-based alloys a mixed effect appears: the dislocation creation with martensite stabilization. In NiTi the effect is mainly related with dislocation creation. In the bridge requirements, in one big storm, the platform and the cables can oscillate several days under the wind action at frequencies close to 1 to $3 \mathrm{~Hz}$ (in the Iroise bridge, figure 1) or 18 $\mathrm{Hz}$ in the St Nazaire bridge [3]. As a matter of fact, after installation, the external action can produce several sets of big number of oscillations separated by lengthy pauses i.e., hours, days or weeks of "good weather" or temporary's intervals of calm without winds inside the storm.

In this work, performed in the frame of SMARTeR ESF, the requirements established by the project centered in the damping of stayed cables for bridges are practically accomplished for cables oscillating at lower frequencies (Iroise bridge at 1 and $3 \mathrm{~Hz}$ ) and, also at higher frequencies as, for instance, the St Nazaire bridge $(18 \mathrm{~Hz})$. The analysis carried out establishes that the life to fracture can be greatly extended by cycling at low stresses as usual in classical material. This condition also imposes to work at a reduced deformation, which diminish the damping capacity. Thus some kind of compromise must be found between the life to fracture and the damping efficiency. It was also found that the hysteresis and the fracture life can be increased by long time aging at $373 \mathrm{~K}$ (fracture at one or two millions of cycles). The temperature effects are studied for 8 percent of deformation and for lower deformation, the analysis for lower deformation and faster cycling indicates that the self heating effects does not affect the hysteresis width at fast cycling. Therefore the main part of the hysteresis at lower frequencies is associated to thermal effects in transformation and retransformation. The behavior of the cables without and with SMA wire/s is studied in the cable 1 of the ELSA facility. In addition, simulations are performed using ANSYS, including an appropriate SMA routine specially developed for this purpose.

\section{Damping of stayed cables in bridges by SMA}

The study is focused in the required properties and performance of the SMA damper for their applications in the Iroise bridge (figure 1-left). In particular, the NiTi wires (SAES getters) are used for the higher number of working cycles only in traction (no compression or bending). The first studies were carried out in the cable number 1 in the ELSA facility, EU-Ispra, Italy (figure 1-right). The experimental analysis is centered in the fatigue-fracture and in the creep behavior. The increase of fatigue-life is partially analyzed in terms of low temperature aging. The SMA creep can be avoided by using the material after appropriate training where most part of the creep has already taken place. For instance, 100 cycles at $8 \%$ at $0.01 \mathrm{~Hz}$ were used. The increase of length ( 1 to $2 \%$ ) can be included in the practical length of the wires of SMA. In fact, the supplementary working cycles, practically, does not induce more increase of length.
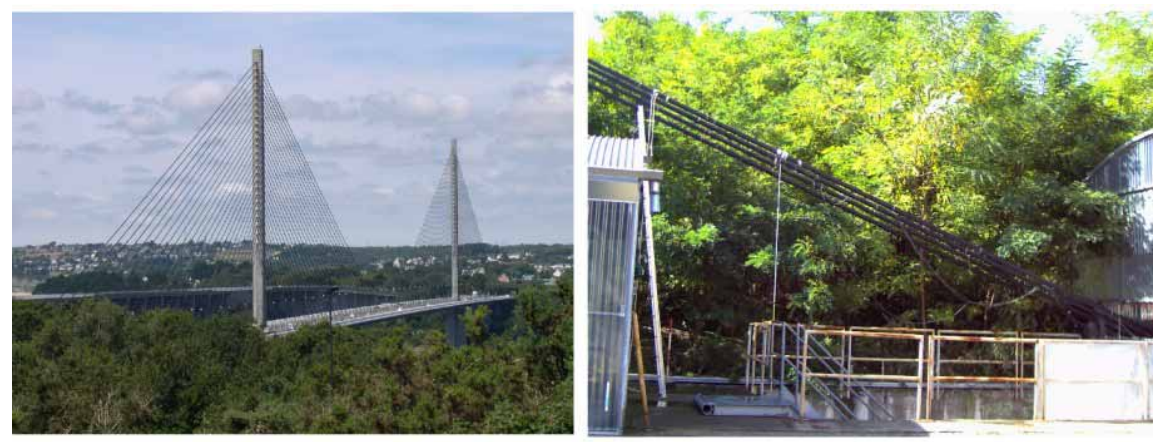

Fig. 1. Targets of the SMA dampers. Left: The Iroise bridge. Right: Cables and sensors in the ELSA facility.

\subsection{Fatigue, fracture and SMA creep}

The experimental analysis is performed using several samples of NiTi from SAES getters (former Special Metals, USA) of similar length (near $200 \mathrm{~mm}$ ) and diameter $2.46 \mathrm{~mm}$. The number of cycles to fracture is highly 
dependent on the stress (figure 2 left). For stresses remaining below $200 \mathrm{MPa}$ the number of cycles to fracture can overcome 1 or 2 million. These conditions can be obtained using lower deformation (under $2 \%$ ) and, eventually long time aged materials that induce higher transformation temperature. In the practical use of the material as a damper is, probably advantageous to use the two types of wires in parallel arrangements, for instance "as furnished" and long time aged. Built the damper with a mix set of wires can be suggested as one more efficient combination for good performance either in summer or winter. In summer the main conversion of mechanic energy into heat is taken by the aged wires and, in winter, the main action is carried out by the "as furnished" wires. In fact, it is possible that the aged wires remains partially in martensite that impedes the conversion of mechanical energy in heat. The standard behavior of the creep in the first 10000 cycles is presented in figure 2 (right). Producing 100 first training cycles is a good way to avoid important creep for subsequent cycles at similar or lower deformations (for stresses no overcoming $600 \mathrm{MPa}$ ). In general, the creep after 10000 cycles remains constant.
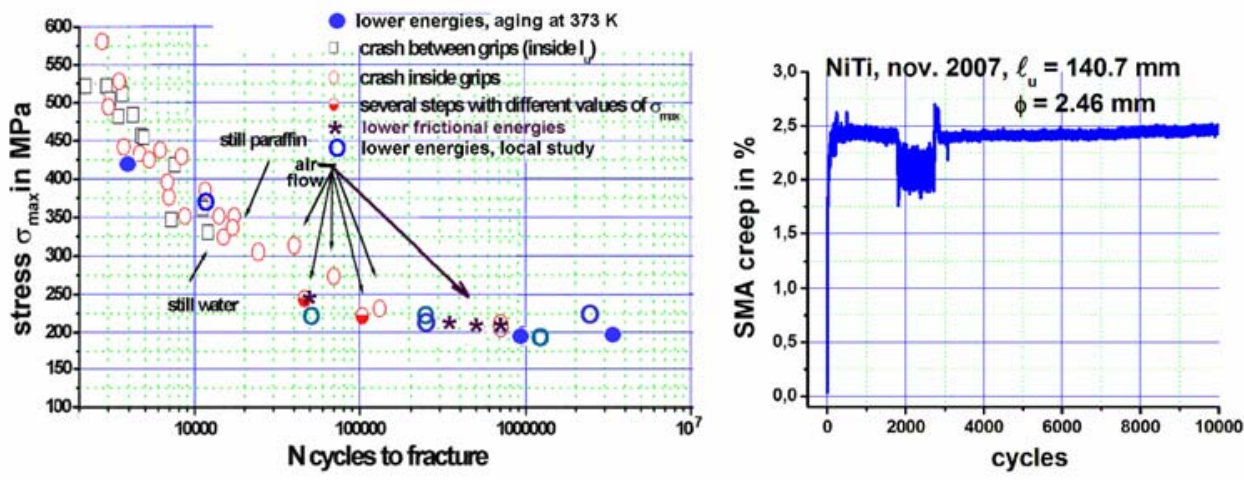

Fig. 2. Left: Evolution of the fatigue-life for NiTi (2.46 mm of diameter) for different applied stresses. Right: SMA creep evolution against the number of cycles.

\subsubsection{Temperature effects}

The temperature effect is related to the external room temperature (summer -winter) or the self-heating associate to cycling. The temperature is evaluated using a K-thermocouple mechanically fixed in the "center" of the sample when a fan is directed to the working space in the MTS. The experimental results, using this rough approach, show changes by a coefficient close to 1.5-2.0 (see, figure 3-left). When the frequency increases from $\mathrm{A}\{0.05 \mathrm{~Hz}\}, \mathrm{B}\{0.5 \mathrm{~Hz}\}, \mathrm{C}\{1.0 \mathrm{~Hz}\}, \mathrm{D}\{2.0 \mathrm{~Hz}\}, \mathrm{E}\{3.0 \mathrm{~Hz}\}$, at $8 \%$ of deformation the temperature increases progressively by the effect of the increased number of hysteresis cycles per second. When the deformation is reduced to $1.25 \%$ from $\mathrm{f}\{0.05 \mathrm{~Hz}\}, \mathrm{g}\{0.2 \mathrm{~Hz}\}, \mathrm{h}\{0.5 \mathrm{~Hz}\}, \mathrm{i}\{1.0 \mathrm{~Hz}\}, \mathrm{j}\{2.0 \mathrm{~Hz}\}, \mathrm{k}\{4.0 \mathrm{~Hz}\}$ and $1\{8.0 \mathrm{~Hz}\}$ to $\mathrm{m}\{16 \mathrm{~Hz}\}$ the self-heating is drastically reduced, at $16 \mathrm{~Hz}$ the mean temperature increases by $7-8 \mathrm{~K}$, which is equivalent to about $50 \mathrm{MPa}$ according to the Clausius-Clapeyron equation (see, figure 3-right). At lower frequencies the temperature fluctuates within a range of $10 \mathrm{~K}$ around the room temperature. This effect represents $\pm 63 \mathrm{MPa}$ of stress. In figure 4 left the hysteresis width is close to $180 \mathrm{MPa}$, subtracting $2 \times 63 \mathrm{MPa}$ the residual hysteresis width is close to that observed in figure 4 right.
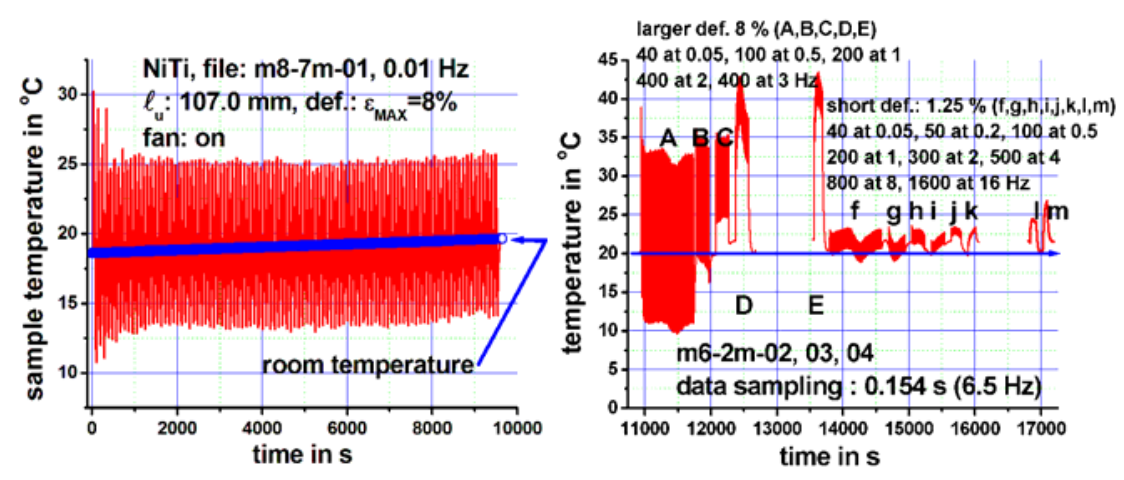

Fig. 3. Left: $\mathrm{NiTi}(2.46 \mathrm{~mm}$ of diameter) temperature in the sample $\mathrm{m} 8$ for the 100 first cycles at $8 \%$. Right: Temperatures for measurements at $8 \%(\mathrm{~A}, \mathrm{~B}, \mathrm{C}, \mathrm{D}$ and $\mathrm{E}$ at respectively $0.05,0.5,1,2$ and $3 \mathrm{~Hz}$ in the sample m6. Series of cycles at 1.25 $\%$ f-g-h-i-j-1-m at, respectively, $0.05,0.2,0.5,1,2,4,8$ and $16 \mathrm{~Hz}$. 


\subsubsection{Cycling effects}

The cycling frequency modifies the aspect of the hysteresis cycles (i.e., figure 4, left and right). These modifications are related to intrinsic changes in the material as, for instance the changes between the first cycle and the cycle $100^{\text {th }}$ (figure 4 left) that generates relevant effects in the SMA creep and an important decrease of the transformation stresses. In addition the hysteresis width diminishes when the cycling frequency increases. This can be attributed to local the heat dissipation and the relative position of the austenite-martensite interface to the temperature front. This is, when the transformation interface tracks the temperature or the inverse situation when the temperature front remains delayed with the position of the transformation interface.
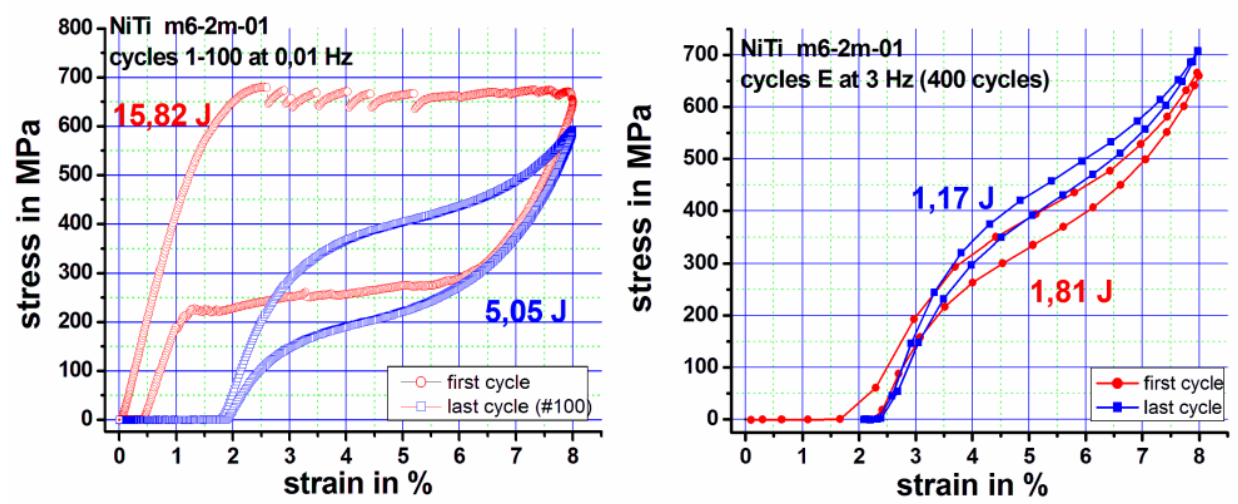

Fig. 4. Evolution in the first set of cycles $(0.01 \mathrm{~Hz})$ and of the $\mathrm{E}$ set of cycles $(3 \mathrm{~Hz})$ at $8 \%$ of maximal deformation. Left: The change on the hysteresis cycle includes near $2 \%$ of SMA creep. The hysteresis width of the last cycle is close to 180 MPa. Right: First (1.81 J) and last (1.17) cycles of the 400 cycles in the E set. The action realized by the sets of A, B, C, D, E and $\mathrm{F}$ create an increase in SMA creep of $0.4 \%$. The hysteresis in the E cycles remains near $60 \mathrm{MPa}$.

The first situation, obtained at slowly cycling frequency, increases the hysteresis cycle by, for instance $\pm 60 \mathrm{MPa}$. However at higher cycling frequency the hysteresis is reduced to their basic width as shows the figure 4 right. It can be seen in figure 5 left the progressive reduction of dissipated energy (hysteresis area) induced by progressive increase of cycling frequency for $8 \%$ of deformation. The measurements show an asymptotic behavior of the dissipated energy towards $1 \mathrm{~J}$ at high frequencies. The figure 5 right indicates the evolution of the energy for lower deformation $(1.25 \%)$. In figure 5 right, the progressive decrease of the dissipated energy for frequencies overcoming $8 \mathrm{~Hz}$ is related to a poor response of the hydraulic system of the MTS machine, giving artificially a smaller hysteresis.
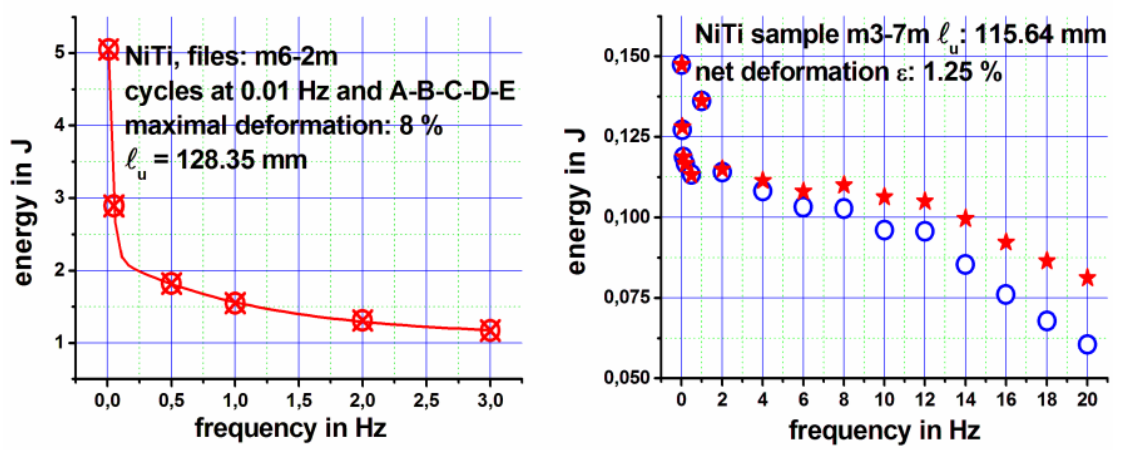

Fig. 5. Left: Evolution of the hysteresis energy for series of measurements at $8 \%$ of deformation at $0.01 \mathrm{~Hz}$ and $\mathrm{A}, \mathrm{B}, \mathrm{C}, \mathrm{D}$, $\mathrm{E}($ at $3 \mathrm{~Hz}$ ) Right: Evolution of the energy for $1.25 \%$ of deformation at progressively higher frequencies (dots). The starts include a linear reduction of deformation induced by inherent artifacts in the MTS equipment.

The figure 6 shows a parabolic behavior of the dissipated energy with the amount of deformation (under $3 \%$ ) and a frequency of $0.05 \mathrm{~Hz}$. The measurements correspond to the calibration part for the realistic experiment focused in damping for the cable 1 in the ELSA facility (Joint Research Center - EU, Ispra, Italy). The available length used was $\ell_{\mathrm{u}}=4.14 \mathrm{~m}$ and the energies were calculated from the experimental cycles 
performed with this length. As shows the figure 6, one linear correction, as the starts in figure 5 right, is probably insufficient for one complete evaluation of the hysteresis effect.

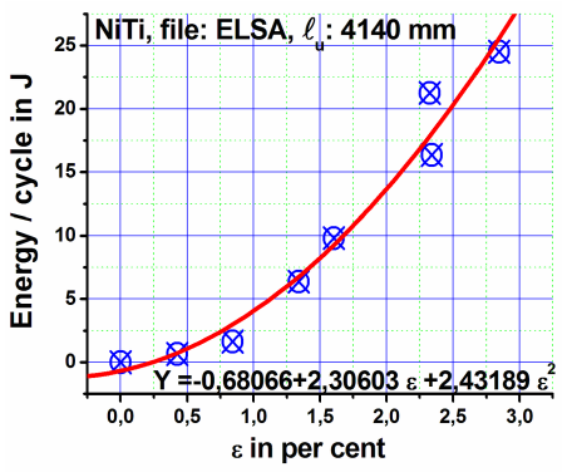

Fig. 6. Curve of the dissipated energy in the hysteresis cycles, at lower deformation using a NiTi wire of $4.14 \mathrm{~m}$, as a function of the transformation strain. The experimental results are fitted by one quadratic form for lower cycling frequency (i.e., near $0.05 \mathrm{~Hz}$ ).

\subsubsection{The aging at $373 \mathrm{~K}$}

Long time aging treatments were done at $373 \mathrm{~K}$. The figure 7 shows the evolution of the stress-strain (or f-x) cycles for different aging times at $373 \mathrm{~K}$, for instance "as furnished" or 0 months, (left), 2 months (centre) and 7 months (right), respectively. The study of more samples in the intermediate times is coherent with the main results. As a reference the $4 \%$ of deformation is used. After two months of aging, an initial increase in the transformation stress in the first cycle and in the $100^{\text {th }}$ cycle in the retransformation stress, near 50 and $20 \mathrm{MPa}$ respectively and, later, after 7 months of aging, a decrease of the transformation and retransformation stress decreases near 40 and $120 \mathrm{MPa}$ in the comparison between the $1^{\text {st }}$ and $100^{\text {th }}$ respectively relative to the sample "as furnished".
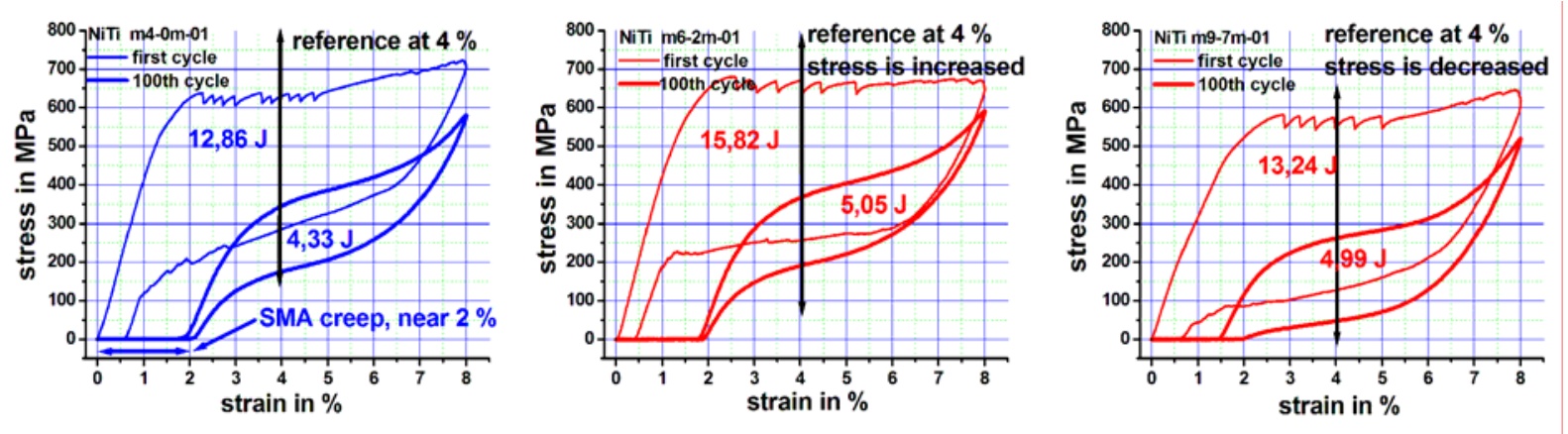

Fig. 7. NiTi, first and $100^{\text {th }}$ cycle at $0.01 \mathrm{~Hz}$ for several aging times at $373 \mathrm{~K}$. Left: as furnished. Centre: 2 months of aging. Right: 7 months of aging

The general observation of the long time aging effects establishes that the behavior of the NiTi is progressively modified in stress-strain by two competitive and apparently opposite actions. For two months of aging, an increase of stress can be observed. However, the more intense effect but opposite to the just mentioned one appears clearly after 7 months of aging, the first complete cycle is displaced to lower stresses and the hysteresis width is increased (at the $4 \%$ of deformation) from 300 to $400 \mathrm{MPa}$. This change of behavior with the aging time is not clear yet and could be related with the growth of Ni rich precipitates.

\section{Experimental results in damping the cable 1 (ELSA) and in modeling}

For a practical control of the SMA in damping for stayed cables several measurements were carried out with the cable no 1 of the ELSA laboratory facility. Only one wire of NiTi SMA was used (2.46 mm of diameter and, after training, $4.14 \mathrm{~m}$ of length). The analysis is devoted to determine the oscillations induced by external periodical forces (roughly 49, 98 and $196 \mathrm{~N}$ ) at the resonance frequency 1.8 and $2.05 \mathrm{~Hz}$. When the SMA is included several values of pre-stress are tested. The maximal cable oscillations, for instance $\pm 80 \mathrm{~mm}$, are 
equivalent to deformations below $3.9 \%$ in the SMA wire system. When the oscillations produce a deformation below $0.5 \%$ in the SMA system the martensitic transformation is not induced and no dissipation of energy take place. The analysis is performed without and with the SMA (see figure 8) [5].
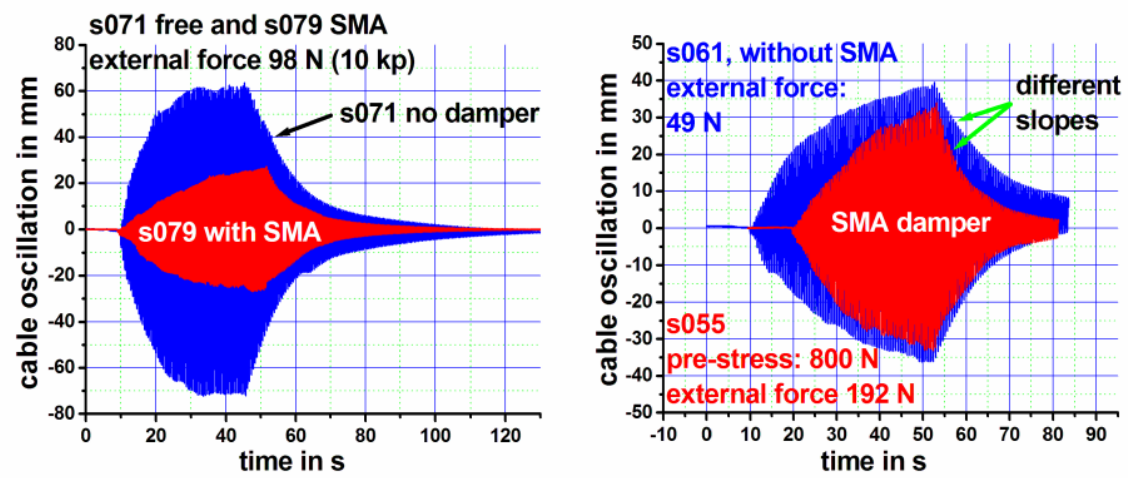

Fig. 8. The cable displacements. Left: equal excitation without and with SMA damper. Similar oscillations for loads of 49 and $192 \mathrm{~N}$. The results show an increased slope with SMA.

The action of SMA reduces the maximal amplitude to 1/3. The study of their behaviour via ANSYS and proprietary routine for the SMA behavior is coherent with the experimental results. Using the available data for the cable 1 an ANSYS simulation can be attained. The cable 1 is filled with wax that produces a relevant "self damping". Figure 9 shows some results obtained by simulation. The figure 9-left shows the response (without and with the SMA) to one Heaviside step in the applied force to the cable. In the right part, the figure visualizes results associated to a cable without damper when is excited by a sinusoidal force varying between 0 and $100 \mathrm{~N}$ at a frequency of $1.81 \mathrm{~Hz}$ for the free cable and exciting at $2.04 \mathrm{~Hz}$ when a damper is attached. After $40 \mathrm{~s}$ of excitation, the force is released and the cable oscillates freely for another $40 \mathrm{~s}$. The experimental results and the simulation show the relevant and clear effects of damping of the SMA in stayed cables.
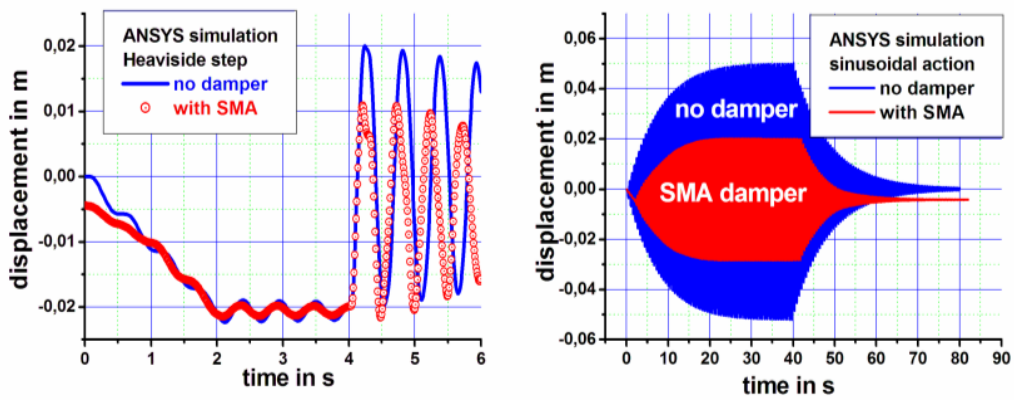

Fig. 9. ANSYS simulation. Left: effect associate to one Heaviside step. Right: effects of a sinusoidal input.

\section{Acknowledgment}

The authors are grateful to C. Auguet (CIRG-DFA-UPC) for their support in the study of the aging in NiTi and to Pablo Riquelme of CAB for his support in the stress-strain measurements performed in NiTi. The work is realized in the frame of SMARTeR project (ESF and MICINN), the financial support is gratefully acknowledged.

\section{References}

[1] V. Torra, A. Isalgue, F. Martorell, P. Terriault, F.C. Lovey, Eng. Struct. 29 1889(2007)

[2] F.C. Lovey, V. Torra Progr. Mater. Sci. 44 (3) 189 (1999)

[3] L. Dieng, M. Peigney, private communication (2009)

[4] J.I. Kim, S. Miyazaki, Acta Mater. 534545 (2005)

[5] V. Torra, A. Isalgue, C. Auguet, G. Carreras, F.C. Lovey, H. Soul, and P. Terriault, JMEPEG_ASM

Internacional, DOI: 10.1007/s11665-009-9442-6, May-2009 